\title{
Determinación de la Relación de Dispersión de un Cristal Unidimensional con el Modelo de Kronig Penney Utilizando Funciones de Green
}

\author{
Luis Alonso Domínguez Villanueva ${ }^{1}$ y Alejandro Galo Roldán ${ }^{2}$ \\ ${ }^{1}$ Escuela de Física - UNAH, mail: villanuevadovi@gmail.com \\ ${ }^{2}$ Escuela de Física - UNAH, mail: alejandrogaloroldan@gmail.com
}

Recibido: 10 de febrero de 2016 / Aceptado: 1 de mayo de 2016

\begin{abstract}
Resumen
Green's functions is applied to describe general qualitative aspects of the band structure for the model Kronig Penney, while equation Lippmann-Shwinger arises as an alternate form of the equation of Schrödinger to solve this problem and especially its solution in a dimension for periodic potentials modeled by Dirac delta, thus deducting the dispersion relation.
\end{abstract}

Keywords: Green function, Lippmann-Schwinger equation, equation Schrödinger, Dirac delta, dispersion relation.

\begin{abstract}
Las funciones de Green es aplicada para describir aspectos cualitativos generales de la estructura de bandas para el modelo Kronig Penney, al mismo tiempo se plantea la ecuación de Lippmann-Shwinger como forma alterna de la ecuación de Schrödinger para la resolución de este problema particular y su solución en una dimension para potenciales periódicos modelados por deltas de Dirac, deduciendo de esta forma la relación de dispersión.
\end{abstract}

Palabras clave: función de Green, ecuación de Lippmann-Shwinger, ecuación de Schrödinger, deltas de Dirac, Relación de dispersión.

\section{INTRODUCCIÓN}

$\mathrm{M}$ UCHAS propiedades de la materia a nivel atómico son determinadas utilizando la ecuación de Schrödinger, debido a ello, para un sistema de electrones en un cristal monodimensional con potenciales periódicos fue estudiada anteriormente por Kronig y Penney [4] en un principio mediante la solución de la ecuacion de Schrödinger no relativista proporcionando soluciones estacionarias bajo una aproximación de los potenciales utilizando deltas de Dirac.

Actualmente el uso de este modelo a nivel pedagógico ha sido muy aplicado ya que presenta un procedimiento analítico para determinar la ecuación de dispersión.

En este artículo se presentará una forma alterna de poder calcular la relación de dispersión, pero es menester mencionar que ya se ha trabajado este mismo problema en 1D por otros métodos diferentes al planteado originalmente por Kronig-Penney como ser: Utilizando transformadas de Laplace [6], método de las matrices de transferencia [2], [1].

El método de la función de Green en 1D para calcular la relación de dispersión será planteado y resuelto de tal forma que se presentará un método alterno para este caso particular en 1D. Por lo tanto la estructura de este artículo será de la siguiente manera: En la sección II se presentarán los conceptos generales sobre la ecuacion de Schrödinger y la función de Green, en la sección III se presentará el desarrollo de la función de Green y en la sección IV] el desarrollo de la relación de dispersión utilizando las funciones de Green.

\section{Conceptos Generales}

Considere la ecuación de Schrödinger para este problema particular como:

$$
-\frac{\hbar^{2}}{2 m} \frac{d^{2} \psi(x)}{d x^{2}}+V(x) \psi(x)=E \psi(x)
$$

donde el potencial cumple con la condición de periodicidad establecida por la estructura cristalina y $a$ es la constante de la red perteneciente a la estructura del cristal por tanto:

$$
V(x+a)=V(x)
$$

además la solución de la ecuación diferencial de Schrödinger se puede expresar en forma alterna como una ecuación integral:

$$
\psi_{K}(x)=\psi_{K}(x)_{0}+P^{\prime} \int G\left(x, x^{\prime}\right) V\left(x^{\prime}\right) \psi_{K}\left(x^{\prime}\right) d x^{\prime}
$$


donde $K^{2}=\left(2 m / h^{2}\right) E, \psi_{K}(x)_{0}=e^{i K x},\left(2 m / h^{2}\right) V(x)=$ $P^{\prime} V(x)$ y el término $G\left(x, x^{\prime}\right)$ es el núcleo del operador integral conocido como la función de Green.

La solución 3 se puede deducir debido a que se puede expresar la ecuación de Schrödinger como una ecuación de Sturm-Liouville con valores en la frontera definida como:

$$
\mathcal{L} y \equiv \frac{d}{d x}\left(p \frac{d y}{d x}\right)+q y=f(x)
$$

que se satisface en el rango de $x_{1} \leq x \leq x_{2}$ con los valores de frontera definidos en $x_{1}$ y $x_{2}$, es decir, si $p=1$ y $q=\left(2 m / h^{2}\right) E$ y $f(x)=\left(2 m / h^{2}\right) V(x) y(x)$ se tiene claramente la ecuación 1 por lo tanto la función de Green necesita satisfacer:

$$
\begin{aligned}
& \mathcal{L} G\left(x, x^{\prime}\right)=\delta\left(x-x^{\prime}\right), \quad \mathrm{y} \\
& y(x)=\int_{x_{1}}^{x_{2}} G\left(x, x^{\prime}\right) f\left(x^{\prime}\right) d x^{\prime}
\end{aligned}
$$

obteniendo la solución 3 planteada como una ecuación integral conocida como ecuación de Lippmann-Schwinger.

\section{CÁlculo de la Función de Green EN UNA DimENSIÓN}

El primer término de la ecuación 3 es la solución perteneciente al electrón en ausencia del potencial (la función de onda del electrón libre). El segundo término de la ecuación, es el perteneciente al electrón en presencia del potencial arbitrario expresado en términos de la función de Green. Por tanto para calcular la función de Green, se utilizará el método de variación de parámetros en la resolución de la ecuación de Sturm-Liouville, ver [5] , debido a que la solución queda expresada en términos de integrales, se asume que la función de Green tendrá la forma:

$$
\begin{array}{ll}
G_{1}\left(x, x^{\prime}\right)=\psi_{1}(x) h_{1}\left(x^{\prime}\right) & x<x^{\prime} \\
G_{2}\left(x, x^{\prime}\right)=\psi_{2}(x) h_{2}\left(x^{\prime}\right) & x>x^{\prime}
\end{array}
$$

donde $h_{1}=A \psi_{2}$ y $h_{2}=A \psi_{1}$, además la función de Green es continua pero existe una discontinuidad para $\partial G / \partial x$ en $x=x^{\prime}$ que se puede apreciar en la ecuación integral de Green, ecuación 5 .

$$
\begin{array}{r}
\lim _{\epsilon \rightarrow 0} \int_{x^{\prime}-\epsilon}^{x^{\prime}+\epsilon}\left[\frac{d^{2} G\left(x, x^{\prime}\right)}{d x^{2}}\right] d x+\lim _{\epsilon \rightarrow 0} \int_{x^{\prime}-\epsilon}^{x^{\prime}+\epsilon} K^{2} G\left(x, x^{\prime}\right) d x \\
=\lim _{\epsilon \rightarrow 0} \int_{x^{\prime}-\epsilon}^{x^{\prime}+\epsilon} \delta\left(x^{\prime}-x\right) d x
\end{array}
$$

Donde la primera y tercera integral son diferentes de 0 , obteniéndose el valor de $A$, quedando como:

$$
A=\frac{1}{w\left[\psi_{1}, \psi_{2}\right]}
$$

donde $w\left[\psi_{1}, \psi_{2}\right]$ es el Wronskiano de $\psi_{1}(x), \psi_{2}(x)$. Sin embargo la ecuación homogénea $\mathcal{L} \psi(x)=0$ tiene como solución:

$$
\psi_{1}(x)=e^{-i K x} \quad \psi_{2}(x)=e^{i K x}
$$

$\psi_{1}(x)$ Representa la función de onda viajando por la izquierda, $\psi_{2}(x)$ Representa la función de onda viajando por la derecha.

Por lo tanto se puede calcular explícitamente el valor de $A$ quedando como $A=-i /(2 K)$ en consecuencia la función de Green es:

$$
G\left(x, x^{\prime}\right)= \begin{cases}-\frac{i}{2 K} e^{i K\left(x-x^{\prime}\right)} & x>x^{\prime} \\ -\frac{i}{2 K} e^{i K\left(x^{\prime}-x\right)} & x<x^{\prime}\end{cases}
$$

De esta manera se conoce la función de Green en 1D, así es posible realizar el análisis con la ecuación integral Lippmann-Schwinger.

\section{RELACIÓN DE DISPERSIÓN 1D}

Dado que ya se conoce la función de Green y por la ecuación de Lippmann-Schwinger, ecuación 3 la solución general quedará representada en forma integral como:

$$
\begin{aligned}
\psi & =e^{i K x}-\frac{2 m}{\hbar^{2}} \int_{-\infty}^{x} \frac{i}{2 K} e^{i K\left(x-x^{\prime}\right)} V\left(x^{\prime}\right) \psi\left(x^{\prime}\right) d x^{\prime} \\
& -\frac{2 m}{\hbar^{2}} \int_{x}^{\infty} \frac{i}{2 K} e^{i K\left(x^{\prime}-x\right)} V\left(x^{\prime}\right) \psi\left(x^{\prime}\right) d x^{\prime} \\
& =\lim _{\epsilon \rightarrow 0} \int_{x^{\prime}-\epsilon}^{x^{\prime}+\epsilon} \delta\left(x^{\prime}-x\right) d x
\end{aligned}
$$

Como se quiere modelar los potenciales que producen cada núcleo del átomo, utilizando las deltas de Dirac y existiendo una periodicidad se tiene que el potencial se puede expresar como:

$$
\frac{2 m}{\hbar^{2}} V(x)=P^{\prime} \sum_{n=-\infty}^{\infty} \delta(x-n a)
$$

Si se incorpora este potencial en la ecuación 8 y dado que la sumatoria es con respecto a $n$ se observa que la solución de las integrales se puede resolver con facilidad gracias a las bondades que nos brinda la delta de Dirac quedando: 


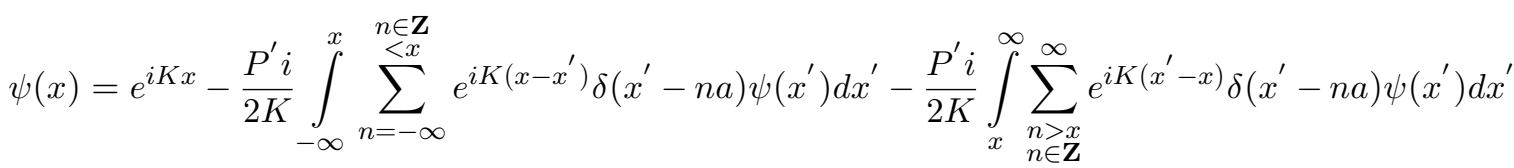

$$
\begin{aligned}
& \psi(x)=e^{i K x}-\frac{P^{\prime} i}{2 K}\left[\sum_{n=-\infty}^{\substack{n \in \mathbf{Z} \\
<x}} e^{i K(x-n a)} \psi(n a) u(x-n a)-\sum_{\substack{n>x \\
n \in \mathbf{Z}}}^{\infty} e^{i K(n a-x)} \psi(n a) u(-x+n a)\right]
\end{aligned}
$$

Donde $u$ es la función escalón unitario

Hasta este momento se tiene resuelta la ecuación de Schrödinger bajo el potencial descrito anteriormente, es decir, las infinitas sumas de deltas de Dirac que modelan el cristal unidimensional.

Pero si se estudian detenidamente los componentes I) y II) del resultado 10 se observa que cada una de estas series producen infinitos términos, más aún, si se estudia el resultado en cualquier celda unitaria el resultado sería muy parecido, cada uno de los términos de la serie aporta parte de información al resultado final; por ejemplo al querer obtener información del comportamiento de esta solución en la celda unitaria cercana al origen en la serie I) para cada valor de $n \in \mathbf{Z}^{-}$, se obtiene información de la celda unitaria y de otras celdas, es decir, si $n=0$ el término $e^{i K x} \psi(0)$ es valido para todo $x>0$, por lo tanto es parte de la solución la celda elegida y todas las celdas unitarias a la derecha de esta. cuando $n=-1$ el término $e^{i K(x+a)} \psi(-a)$ es valido para todo $x>-a$ donde también es parte de la solución de la celda unitaria cercana al origen, y además es parte también de la solución todas las celdas a la derecha. Así sucesivamente se observa que cada término de la serie I) y II) produce parte de la solución en la celda que se escoje. ver figura 1 y 3 .

$$
\begin{gathered}
\text { Para I) } \sum_{\substack { n=-\infty \\
\begin{subarray}{c}{n \in \mathbf{Z} \\
<x{ n = - \infty \\
\begin{subarray} { c } { n \in \mathbf { Z } \\
< x } }\end{subarray}} e^{i K(x-n a)} \psi(n a) u(x-n a) \\
\text { Para II) } \sum_{\substack{n>x \\
n \in \mathbf{Z}}}^{\infty} e^{i K(n a-x)} \psi(n a) u(-x+n a)
\end{gathered}
$$

Se puede obtener la relación de dispersión si se estudia una de las celdas unitarias solamente con la presencia del potencial existente en ella, y se justifica debido que la utilización del teorema de Bloch [3] proporciona la solución en cualquier otra celda unitaria. Por lo tanto si se toma la celda unitaria cercana al origen con la presencia de los potenciales existentes en ella y de la solución 10 la función $\psi(x)$ en esta celda quedaría expresada como:

$$
\psi(x)=e^{i K x}-\frac{P^{\prime} i}{2 K}\left[e^{i K x} \psi(0)+e^{i K(a-x)} \psi(a)\right]
$$

Al utilizar las condiciones de frontera,

$$
\begin{aligned}
& \psi(0)=1-\frac{P^{\prime} i}{2 K}\left[\psi(0)+e^{i K a} \psi(a)\right] \\
& \psi(a)=e^{i K a}-\frac{P^{\prime} i}{2 K}\left[e^{i K a} \psi(0)+\psi(a)\right]
\end{aligned}
$$

Ordenando:

$$
\begin{aligned}
1 & =\left(1+\frac{P^{\prime} i}{2 K}\right) \psi(0)+\frac{P^{\prime} i}{2 K} e^{i K a} \psi(a) \\
e^{i K a} & =\frac{P^{\prime} i}{2 K} e^{i K a} \psi(0)+\left(1+\frac{P^{\prime} i}{2 K}\right) \psi(a)
\end{aligned}
$$

por tanto la solucion de este sistema de ecuaciones lineales es:

$$
\begin{aligned}
\psi(0) & =\frac{\beta-\alpha e^{2 i K a}}{\beta^{2}-\alpha^{2} e^{2 i K a}} \\
\psi(a) & =\frac{(\beta-\alpha) e^{i K a}}{\beta^{2}-\alpha^{2} e^{2 i K a}}
\end{aligned}
$$

Donde: $\alpha=\left(P^{\prime} i\right) /(2 K)$ y $\beta=1+\alpha$. Utilizando las condiciones de frontera con el teorema de Bloch se tiene $\psi(a)=\psi(0) e^{i k a}$ entonces:

$$
e^{-i k a}=\cos K a+\frac{P}{K a} \sin K a-i \sin K a
$$

donde $k$ es el vector de onda del espacio recíproco o espacio de Fourier y $P=P^{\prime} a$. Por tanto igualando parte real con parte real en la igualdad 12 se obtiene la relación de dispersión.

$$
\cos k a=\cos K a+\frac{P}{K a} \sin K a
$$

Por lo tanto se ha presentado la resolución de la relación de dispersión por el método de la funciones de Green, 


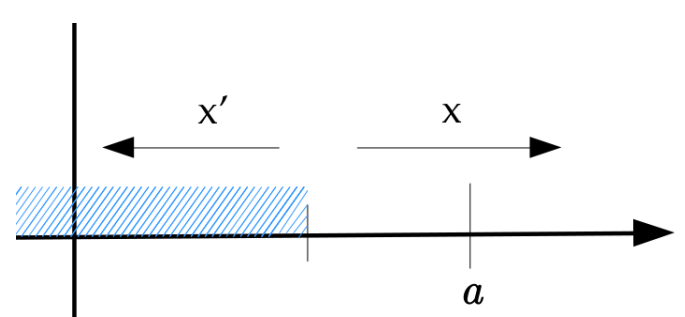

Figura 1: Esquema de valor permitidos de $x>n$

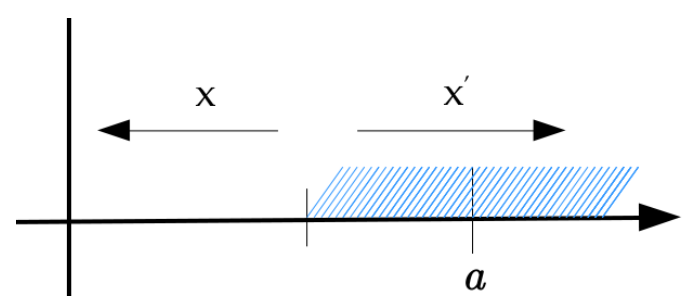

Figura 3: Esquema de valor permitidos de $x<n$

aunque en la literatura el método de la función de Green con la ecuación de Lippmann-Schwinger es presentado para la resolución de problemas de dispersión y el análisis de estructuras de bandas, ha servido de utilidad para la resolución del modelo Kronig Penney.

\section{Conclusiones}

1. Se utilizó un herramienta matemática para poder calcular la relación de dispersión concerniente a las bandas de energía permitidas y prohibidas utilizando las funciones de Green, obteniendo el mismo resultado planteado por otros autores [4, [6], [1].

2. El estudio de las estructuras cristalinas mediante la ecuación de Schrödinger es abordado mediante el teorema de Bloch y las condiciones de frontera, quedando claro que sin esta herramienta sería difícil calcular la relación de dispersión como se ha hecho actualmente.

\section{REFERENCIAS}

[1] Beltran, J.H.V. (2013). Método Generalizado de la Matriz de Transferencia (MGMT); Método de las Funciones de Green de Superficie (MFGS), Relaciones y

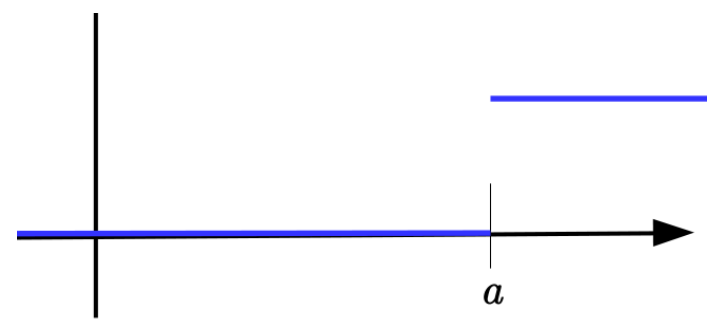

Figura 2: Esquema de la función escalos unitario $u(x-$ a)

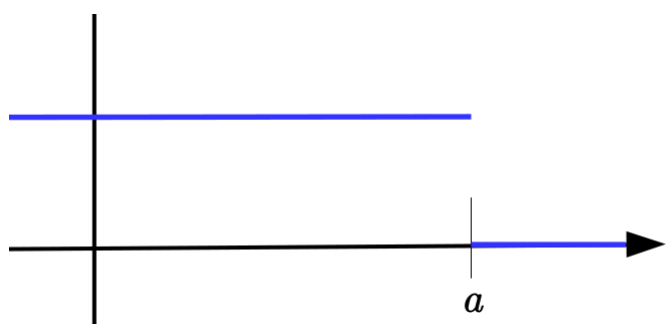

Figura 4: Esquema de la función escalón unitario $u(-x+a)$

Aplicaciones en Sistemas Semiconductores Periódicos. Tesis, Universidad Nacional de Colombia Facultad de Ciencias, Departamento de Física Bogota, Colombia.

[2] Griffiths, D.J. (2005). Introduction to quantum mechanics. Pearson Prentice Hall, New Jersey, 3nd enlugar. ISBN 0131118927.

[3] Kittel, C. (1997). Introducción a la Física del Estado Sólido. Wiley, Toronto, 3 a, spañol enlugar. ISBN 8429143173.

[4] Kronig, R. y Penney, W., Quantum mechanics of electrons in crystal lattices. En Proceedings of the Royal Society A: Mathematical, Physical and Engineering Sciences, Volumen 130 (1931) (814): 499-513. ISSN 1471-2946. doi:10.1098/rspa.1931.0019. URL http://dx.doi.org/10.1098/rspa.1931.0019

[5] Nagle; Saff y Snider (2005). Ecuaciones Diferenciales y problemas con valores en la frontera. Pearson Educacion, 4th enlugar. ISBN 970-26-0592-X.

[6] Santana, P.H.A., Use of the Laplace transform method to solve the one-dimensional periodic-potential problem. En Am. J. Phys., Volumen 41 (1973) (10): 1138. ISSN 0002-9505. doi:10.1119/1.1987504. URL http://dx.doi.org/10.1119/1.1987504 